\title{
The TCM Research Status of Macular Epiretinal
}

\author{
Xuanlin Zhao ${ }^{1}$, Xiaoqin Lei ${ }^{2}$ ** \\ ${ }^{1}$ Shaanxi University of Chinese Medicine, Xianyang 712046, Shaanxi, China \\ ${ }^{2}$ Xi' an Fourth Hospital, Xi'an 710004, Shaanxi, China \\ *Correspondence Author
}

\begin{abstract}
Macular epiretinal membrane belongs to the category of "blurred vision and deformation vision" in traditional Chinese medicine. In recent years, based on the experiences and viewpoints of modern and ancient physicians, this article reviews the etiology and pathogenesis, syndrome differentiation, staging and classification, traditional Chinese medicine treatment, acupuncture treatment, and traditional Chinese medicine treatment for postoperative complications of macular epiretinal membrane.
\end{abstract}

Keywords: Macular epiretinal membrane, Traditional Chinese Medicine, Review.

\section{Introduction}

The anterior macular membrane (MEM) is a disease located between the inner retinal boundary membrane and the vitreous membrane, with the fiber membrane as the main lesion[1]. Whether MEM is secondary to other ocular diseases can be divided into two categories: idiopathic macular epiretinal (IMEM) and secondary macular epiretinal. IMEM refers to the patient generally without other eye disease and the macular area, the secondary macular anterior membrane refers to the macular epiretinal secondary to other eye diseases, such as ocular inflammation, vascular lesions, diabetic retinopathy, intraocular surgery or other eye diseases[2]. The survey showed an incidence of preretinal membranes of $18.5 \%$. The incidence rates over 50 years reached $5.3 \%-18.5 \%$ and $35 \%$ in patients aged 70 to 79 , of whom 1.7\%-6.2\% were IMEM[3-7]. The incidence of this disease was reported as ranging from 5.5\%-12.0\%. A positive association of the disease with age was seen. At present, in the early stage of the macular epiretinal, western medicine mainly takes observation without special treatment. At this stage, there are no specific drugs or effective treatment means. When the anterior membrane pulls the retina and has a great impact on vision, the main treatment is surgery. However, there is no conclusion on the choice of surgical timing, and for different patients, different surgical timing also has different effects on the prognosis of MEN. And there are some anterior macular membrane recurrence after Bolivia, there are such as macular edema, lens opacity and other postoperative complications. The treatment of this disease in traditional Chinese medicine is different from western medicine, and the treatment methods are relatively many. For the macular epiretinal, the non-surgical treatment and postoperative complications of traditional Chinese medicine have unique advantages compared with western medicine.

\section{The Traditional Chinese Medicine Disease Machine}

Traditional Chinese medicine believes that the macular epiretinal is an internal barrier, which belongs to the category of vision and vision, the liver is open to the eye and the eye is outside the liver, so the disease is sufficient liver blood, and whether the liver qi is closely related. The five-round theory in the Huangdi Neijing believes that macular can be attributed to the pupil god, and the five elements of the pupil god correspond to the water and five zang corresponding to the kidney, so it is hidden as the kidney. In addition, the macular is yellow, yellow corresponds to the spleen soil in the five elements, and the macular multiple edema, similar to the characteristics of the spleen like dryness and dampness, so the macular disease is more responsible for the spleen soil. Therefore, the occurrence of pathogenesis is closely related to the dysfunction of the viscera like liver, spleen and kidney[8]. As for internal barriers, "Ling shu · pulse" mentioned: "kidney foot Yin veins, move is sick, as seen... less Yin disease as seen, Yin invasion... this house is dizzy." It can be seen that the disease is not only closely related to the kidney, but also related to the adequacy of Yin fluid and room labor injury. Jin Dynasty Liu Hejian put forward: "XuanFu, nothing is not... is the gas up and fall of the portal... XuanFu occlusion, and gas blood, health spirit, can not rise and out so, each with the little..." from Liu Hejian point of view, Xuanfu for the air machine rise important portal, XuanFu closed can directly lead to fine micro matter can not be noted in the eye, leading to the occurrence of disease, the emergence of blurred vision decline[9]. Highlight the importance of kidney Yang in the disease, kidney Yang is insufficient, can not warm the spleen and stomach, qi and blood should not be biochemical, the liver should not hide blood, visual clothing function is abnormal, leading to the formation of the anterior membrane. Although various doctors currently have their own views on the etiology of MEM, the various views are interrelated and support each other.

To sum up, the related viscera of MEM are mainly liver, spleen and kidney, and the basic pathogenesis is the deficiency of qi and blood. The deficiency of spleen and kidney Yang leads to Xuanwu Fu depression, phlegm and turbidity invasion, and blood stasis.

\section{TCM Therapy}

\subsection{Syndrome Differentiation}

Only by combining dialectical and disease differentiation can dialectical treatment can clinical drug treatment be more effectively, and syndrome differentiation is needed in the treatment of MEM[10]. Other studies showed that the total etiology of the disease is spleen and kidney Yang deficiency, named fire failure, and the main evidence type is spleen and kidney Yang deficiency[11]. Referring to the Standard for the Diagnosis and Efficacy of Traditional Chinese Medicine 
Diseases, etc[12]. And the Ophthalmology of Traditional Chinese Medicine[13], the disease is divided into: liver stagnation, liver and kidney Yin deficiency, qi and blood, phlegm and wet general, Yin deficiency fire type, blood stasis, after the study of 120 IMEM patients found the most common three: stasis, sputum dampness and liver and kidney Yin deficiency; and typical stasis is not common, after macular membrane surgery, due to unavoidable mechanical pull, may lead to a small bleeding, edema, so this type of patients is more common after macular membrane surgery[14]. It is believed that the initial macular area edema with the anterior membrane stage, mostly spleen deficiency water generalization, the middle macular anterior membrane formed a wrinkle stage, mostly blood stasis and sputum coagulation type, and the later stage is mostly liver and kidney deficiency type[9]. It is believed that the common liver and kidney deficiency, Qi and blood deficiency, blood stasis block, phlegm turbidity on the invasion type[15]. Other studies found that after IMEM, patients with blood stasis were the most common, followed by sputum and wet mutual junction and liver and kidney Yin deficiency, and the macular thickness of patients with blood stasis and phlegm junction was significantly greater than that of liver and kidney Yin deficiency.

\subsection{Treatment}

\subsubsection{Needle-drug binding}

Doctors have carried out in-depth exploration on the treatment of MEM, improve the lesion structure, delay the development of the disease, improve the vision, and improve the quality of life[16]. After learning the clinical experience of Mr. Zhang Ren, the use of acupuncture and moxibustion combined with local hot compress of Taoren safflower and other drugs, after 10 days of treatment, the affected eye vision increased from 0.3 to $0.8[17]$. Such as to relieve the liver and spleen as the treatment, the use of "three eyes" combined with Chahu, Yujin, Danpi and other drugs to treat a patient with anterior macular membrane, vision was significantly improved.

\subsubsection{Traditional Chinese medicine treatment}

The treatment of tonifying liver and kidney, qi and blood circulation, removing blood stasis, from the puerroot, astragalus, wolfberry, chicken and blood vine peeling soup treatment of 13 patients with anterior macular membrane, vision significantly improved, but the anterior membrane reduction effect was not good[9]. Chinese wolfberry poria cocos soup was used to treat macular edema after IMEM[18]. The postoperative macular edema was significantly improved and the vision was improved, but the macular volume change did not be significantly different until 3 months after taking the medication[10]. Such as thermotonic from the treatment of patients with early idiopathic macular membrane, control group is oral compound blood plug capsule, medication for two months, in the 1,2, May observation after treatment of the best correction of vision, yellow plate thickness is significantly better than simple control group of promoting blood circulation and remove blood stasis, but weakened advantage over time[19]. A case of idiopathic anterior macular membrane with macular edema was treated before treatment, the best corrected vision of the right eye was 0.3 , the left eye was 1.0, the best corrected visual acuity of the right eye was 1.0 and the left eye after 3 courses of treatment. In the Qing Dynasty, Huangyan mentioned the treatment of internal barriers in "the spleen is the first of the Yin, the eye is the blood, the spleen deficiency is the essence of the five viscera, ignoring the spleen and stomach is to treat the symptoms rather than the root cause, treat the spleen and stomach, and treat the spleen with the foundation." "Review Yao Letter" recorded the treatment of positive and negative evidence, that the disease is Yin and Yang, Yin Sheng Yin, should use fill Yin soup, rise Yang diarrhea Yin soup, should use even tiller benefit Yin pills.

\section{Discussion}

Based on the views of doctors in the past dynasties, the deficiency of liver and kidney and qi and blood are the premise of the occurrence of macular epiretinal, and the physiological characteristics of yellow and its prone to edema are corresponding to the physiological characteristics of the five-color main yellow and like dryness and dampness. Therefore, from the perspective of traditional Chinese medicine, the occurrence of the disease mainly lies in the liver, kidney and spleen[10]. The reuse of cinnamon, which treats the disease with warm tonic, emphasizes the special position of renal Yang in the treatment of macular epiretinal.

Before macular membrane belongs to the category of traditional Chinese medicine, internal obstacle disease course, Chinese medicine soup treatment is effective, but long treatment cycle, and the treatment and adjustment, patients, it is difficult to insist, and some foreign patients cost, will increase the burden of patients, in the search of special prescription, Chinese patent medicine, or research and development of new drugs, or surgery combined Chinese medicine, acupuncture to conquer MEM at the same time, with the help of Chinese medicine ions[20]. Whether the new treatment methods can improve the efficacy and shorten the treatment cycle, in the future research of TCM treatment of macular epiretinal, we should not only further study the treatment of the disease, but also give full play to the advantages of the diversity of TCM treatment methods, multi-pronged, to bring good news to patients.

\section{References}

[1] Liu Jiaqi, Li Fengming. Practical ophthalmology[M]. Beijing: People's Health Press, 2010:448.

[2] Fraserbell S, Guzowwski M, Rochtchina E, et al. Five -year cumulative incidence and progression of epiretinal membranes: The Blue Mountains Eye Study[J]. Ophthalmology, 2003, 110(1): 34-40.

[3] Noda Y, Yamazaki S, Kawano M, et al. Prevalence of epiretinal membrane using optical coherence tomography[J]. Nippon Ganka Gakkai Zasshi, 2015, 119(7): 445-450.

[4] You Q, Xu L, Jonas JB. Prevalence and as sociations of epiretinal membranes in adult Chinese: The Beijing eye study[J]. Eye, 2008, 22 (7): 874-879.

[5] Zhu XF, Peng JJ, Zou HD, et al. Prevalence and risk factors of idiopathic epiretinal membranes in Beixinjing blocks, Shanghai, China[J]. PLoS One, 2012, 7(12): e51445. 
[6] Tang Shibo. Surgery for macular disease[M]. Beijing: People's Health Publishing House, 2005: 191-199.

[7] Gao Jun, Chao Guojun. Effect of addition and subtraction on anterior membrane optic function of early idiopathic macular plaques[[J]. Hebei Traditional Chinese medicine, 2016, 38(10): 152811530.

[8] Wang Yubin, Guo Lianchun, Zhong Ruisheng, Zhuo Nan, Lin Yuan. Clinical observation of 13 macular epiretinals with autologous decidua soup[J]. Grassroots Medicine Forum, 2017, 21(28): 3909-3911.

[9] Chen Li, Ran Qi, Feng Chi. Intervention of early idiopathic macular epiretinal[J]. Clinical Medical Research and Clinical Practice, 2016, 1(12): 98-100.

[10] The syndrome distribution of the anterior macular membrane and the clinical study of traditional Chinese medicine combined with vitrectomy treatment[D]. Chinese Academy of Traditional Chinese Medicine Sciences, 2019.

[11] Terminology of Clinical Diagnosis and Treatment of Traditional Chinese Medicine, National Administration of Technical Supervision, National Standard of the People's Republic of China, Beijing: China Standard Publishing House

[12] TCM Ophthalmology, 1997. Shanghai Science and Technology Publishing House, October 1985, the first edition.

[13] Xiao Wenzheng, Xie Like, Hou Le. One case of anterior retinal macular membrane examination was treated with traditional Chinese medicine[J]. TCM Guide, 2013, 19(04): 89-90.

[14] Zhu Hai, Liang Xianjun, He Jinxian, Chen Shaoji, Lin Yingjie, Yang Xueyan, Zeng Sheng. Clinical analysis of TCM syndrome type and retinal thickness in the macular area after idiopathic anterior macular membrane surgery $[\mathrm{J}]$. New traditional Chinese medicine, 2011, 43(03): 57-59.

[15] Hu Yannian. The idiopathic macular anterior retinal membrane[J]. Fundi Disease, 1990, 6:181

[16] Xing Xiaona, competition from Jin, Zhang Licai, Cong Yuan, Chen Jingtao, Yang Huawei, Ma Lihua, Sun Chunxia. One case of preretinal macular membrane[J]. TCM Guide, 2015, 21(24): 87-88.

[17] Hang Weiqi, Zhang Xianmei, Qin army, Luo Guoping. Needneedle to treat early idiopathic macular epiretinals in one case $[\mathrm{J}]$. Chinese Journal of Traditional Chinese Medicine Ophthalmology, 2017, 27(06): 405-406.

[18] Zhou Shangkun, Qin Hong, Ma Qunying, Tang Youzhi, Wang Huijuan, Wang Lixia.Clinical observation of macular edema after the treatment of macular epiretinal dissection[J]. Chinese Journal of Traditional Chinese Medicine Ophthalmology, 2018, 28(05): 310-313.

[19] Limb GA, Earley O, Jones SE, et al. Expression of mRNA coding for TN F alpha, IL- 1 beat and IL- 6 by cells infiltrating retinal membranes[J]. Graefe Arch Clin Exp Ophthalmol 1994, 232: 646-651. 